\title{
Application Comparison of Closed Reduction with Hollow Screw Internal Fixation and Open Reduction with Special Shaped Plate Internal Fixation in Calcaneal Fracture
}

\author{
L. ZHAI, K. HUANG, B. LIN, Q. GUO, Y. LIU, L. SHEN AND G. MA* \\ Department of Orthopaedics, Tongde Hospital of Zhejiang Province, Hangzhou, Zhejiang310012, China
}

Zhai et al.: Comparison of Closed Reduction and Open Reduction in Calcaneal Fracture

This study article was conducted to comparing the application effect of closed reduction with hollow screw internal fixation and open reduction with special-shaped plate internal fixation in calcaneal fracture. 60 patients with calcaneal fracture admitted to our hospital from April 2015 to April 2019 were selected, who were randomly divided into A group and B group, with 30 cases in each group. The A group was treated with closed reduction with hollow screw internal fixation, while the B group was treated with open reduction with special-shaped plate internal fixation. The operation time and bleeding volume during operation were compared, and the changes of calcaneal tubercle articular angle (Bohler angle) and calcaneal cross angle (Gissane angle) before operation and 3 mo after operation were compared, and the intraoperative and postoperative complications and functional recovery of affected foot were also compared. The operation time in the A group was significantly shorter than that in the B group (p<0.05), and the amount of bleeding during operation in the A group was significantly less than that in the B group $(\mathbf{p}<\mathbf{0 . 0 5})$. The Bohler angle and Gissane angle at 3 mo after operation in the 2 groups were significantly higher than those before operation $(p<0.05)$, and there was no significant difference in Bohler angle and Gissane angle between the $A$ group and $B$ group at 3 mo after operation $(p>0.05)$. There was no nerve injury in the 2 groups during operation, and the incidence rate of total postoperative complications in the A group was significantly lower than that in the B group $(p<0.05)$. All patients were followed up for 3 to $15 \mathrm{mo}$, and there was no significant difference in the excellent and good rate of foot function recovery between the 2 groups at the last follow-up $(p>0.05)$. Closed reduction with hollow screw internal fixation and open reduction with special-shaped plate internal fixation all are satisfactory of reduction of fracture in the treatment of calcaneal fracture, and the function of foot of them all recover well after operation, However, closed reduction with hollow screw internal fixation could shorten the operation time, reduce intraoperative bleeding and reduce postoperative complications.

Key words: Bone screws, calcaneus, recovery of function, postoperative complications, fracture fixation internal

Calcaneal fracture, as a common type of tarsal fracture in clinical practice, takes up nearly 60 to 65 $\%$ of all cases of tarsal fracture. Principally, calcaneal fracture is manifested by the acute pain, swelling and ecchymosis in heal, or the inability to stand up or walk, or the pressing pains in calcaneus, severely disturbing the regular life of patients ${ }^{[1,2]}$. Due to the complexity in the anatomic structure and geometrical morphology of calcaneus and the surroundings, as well as the conditions that calcaneal fracture frequently complicated with the intraarticular displacement, which increases the difficulty of treatment, and deteriorates the prognosis, with the inevitable sequalae and a high

*Address for correspondence

E-mail: bangy97263chu@126.com morbidity $\operatorname{rate}^{[3,4]}$. Currently, surgical replacement remains the major method for treatment of calcaneal fracture, but it is still controversial in selecting the type of the replacement and internal fixation.

In recent $\mathrm{y}$, closed replacement and internal fixation of cannulated screw and surgical open reduction and internal fixation of irregular steel plates are the common methods for clinical treatment of calcaneal fracture, with precise replacement and promising recovery of the foot function ${ }^{[5-7]}$. In this study, we conducted the closed replacement and internal fixation of cannulated screw and surgical open reduction and internal fixation of 
irregular steel plates for calcaneal fracture to compare the incidence of surgical trauma, clinical efficacy and postoperative complications between two groups, so as to provide the reference for clinical treatment.

\section{MATERIALS AND METHODS}

\section{General data:}

A total of 60 calcaneal fracture patients who were admitted to this hospital for treatment between April 2015 and April 2019 were randomized into the Group A and Group B, with 30 patients in each group. In Group A, there were 21 males and 9 females, aged between 18 and $71 \mathrm{y}$ old, with an average of (36.85 \pm 7.42$)$ y old; the duration from the fracture onset to the surgery ranged from $14 \mathrm{~h}$ to $8 \mathrm{~d}$, averaged at $(3.95 \pm 0.61) \mathrm{d} ; 17$ patients had fracture on the left foot, 11 on the right foot and 2 had fracture on both feet; for Sanders type, 19 patients had type II fracture, and 11 had type III fracture; for accident cause, 7 patients had car-accident-caused fracture, 18 had falling-caused fracture and 5 had other types of fracture; for complications, 2 patients had the thoracolumbar burst fracture, 1 had pelvic fracture and 1 had intertrochanteric fracture. In Group B, there were 23 males and 7 females, aged between 20 and $69 \mathrm{y}$, with an average age of (37.26 \pm 7.38$)$ y old; the duration from the fracture onset to the surgery ranged from $16 \mathrm{~h}$ to $7 \mathrm{~d}$, averaged at $(3.91 \pm 0.58) \mathrm{d} ; 15$ patients had fracture on the left foot, 14 on the right foot, and 1 had fracture on both feet; for Sanders type, 18 patients had type II fracture, and 12 had type III fracture; for accident cause, 6 patients had car-accident-caused fracture, 20 had falling-caused fracture and 4 had other types of fracture; for complications, 3 patients had the thoracolumbar burst fracture, and 1 had pelvic fracture. Comparison over the general data of patients between two groups showed no significant difference $(p>0.05)$ and this study had been approved by the Ethical Committee of the hospital.

Inclusion criteria-Patients with fresh, closed, calcaneus fracture; patients who underwent the regular X-ray examination, computed tomography (CT) scanning and 3-dimensional reconstruction; patients who took the symptomatic treatment, including fixation, raising and detumescence of affected limb, and active treatment for the complications of fracture; patients who were informed of the study protocol and agreed to participate in this study.
Exclusion criteria-Patients with open fracture, or dated fracture; patients with ankle fracture or tarsal fracture; patients with the history of ankle injury; patients with the ankle dysfunction in the affected limb prior to the fracture; patients with the pathological fracture due to the primary osteogenic tumor, osteoid lesion, cystic pathogenic damage or bone metastases; patients with diseases in nervous system or mental disease.

Group A-Patients underwent the closed replacement and internal fixation of cannulated screw. Patients were anesthetized in supine position by the combined spinal and epidural analgesia, followed by the sanitization and draping and surgical treatment under the C-type arm. Punctuating point and direction were selected upon the preoperative X-ray examination and CT examination. From the posterior and lower calcaneal tuberosity, a 4.0 Steinmann pin was drilled vertically and flipped to tract to correct the varus and shortening of calcaneus. Then, under the guidance of C-type arm, a guide pin was inserted percutaneously from the calcaneus tuberosity to fix the site of fracture. Later, in the lateral side of the Achilles tendon, a Steinmann pin was drilled on the top of the calcaneal tuberosity, where the pin tip was advanced to the fracture space and then lifted up to support the bone on the articular surface to correct the Bohler angle and Gissane angle, while the bone at the fracture site was fixed by the 1 or 2 pins drilled beneath the lifted pin. Otherwise, 1 or 2 cannulated screws were inserted along the pin, and the pin was then taken out, followed by suture and plaster external fixation.

Group B-Surgical open reduction and internal fixation of irregular steel plates was carried out. Patients were anesthetized in lateral position of the non-affected side by the combined spinal and epidural analgesia. An L-shape incision was made on the lateral calcaneus and in the middle of Achilles's tendon and fibula and the boundary between the dorsal and pedis of foot skin, a longitudinal and horizontal incision was made respectively. Then, the incision of the calcaneocuboid joint slightly leaned against the dorsal side. During the surgery, incision should reach the surface of bone, where the electrotome was only suggested for hemostasis. To expose the tendon of peroneus longus, the tissues from the skin to the periosteal flap were flicked over, and then 3 kirschner wires were inserted into the astragalus to fix the flaps. Thereafter, paries lateralis was open to expose the lower joint surface which was then turned over by $90^{\circ}$, and then the sclerites in collapse were poked to 
restore the Gissane angle. The middle to lower joint surface, if necessary, could be exposed by removing the fat in the tarsal canal, while since the anterior to the lower joint surface was hardly to be exposed, a kirschner wire was inserted transversely through the calcaneus tuberosity and dragged downwards to restore the Bohler angle. Thereafter, along the calcaneal axis, a kirschner wire was used to poke to restore the calcaneus height and then inserted directly into the astragalus for restoration and to widen the calcaneus by suppressing longitudinally the paries lateralis of calcaneus. Under the C-shape arm, Bohler angle was adjusted to $30^{\circ}$ and after the joint restoration, the artificial bone or iliac bone was inserted in the space of calcaneus according to the defect of subarticular surface bone. A suitable, plastic plate was placed on the paries lateralis of calcaneus, where the screw should be inserted into the sustentaculum tali. Finally, the kirschner wires were taken out, with the thorough hemostasis and a drainage tube was inserted, followed by the suture and compressed dressing.

\section{Postoperative treatment:}

After operation, patients in two groups were required to lift their affected limbs and take the antibiotics for 3 to $5 \mathrm{~d}$. Besides, patients were advised to taken the flexion and extension exercise of toes within 24 to 48 $\mathrm{h}$ after operation. For patients in Group B, the drainage tube was removed at 48 to $72 \mathrm{~h}$ after surgery, and the plaster support was removed at 2 to $3 \mathrm{w}$ after surgery. For patients in two groups, regular X-ray examination was carried out after surgery, and they were further required to perform the progressive, enhanced and active functional exercise at $4 \mathrm{w}$ after operation, and then the weight-free functional exercise at 6 to $8 \mathrm{w}$ and the progressive weight-bearing exercise after the reunion of fracture, i.e. 8 to $12 \mathrm{w}$ after operation.

\section{Indicators for observation:}

Operation time and intraoperative bleeding amount; Changes of Bohler angle and Gissane angle before operation and at 3 mo after operation based on the results of X-ray examination and CT examination; intraoperative and postoperative incidence of complications: Incidence rates of complications, including the intraoperative nerve damage, and postoperative wound infection, marginal necrosis of skin and traumatic subtarlar arthritis.

\section{Functional restoration of affected foot:}

After operation, patients participate in the follow-up lasting between 3 and 15 mo and at the final follow-up, Maryland scoring system was utilized to evaluate the restoration of foot function, including pain (45 points), activity (45 points), walking distance (10 points), appearance (10 points), ankle (5 points), stability (4 points), support (4 points), claudication ( 4 points), shoe (10 points), climbing upstairs and downstairs (4 points) and landform (4 points). Results of evaluation were classified as follows: Excellent for patients with score $\geq 90$ points, good for those between 90 and 75 , normal for those between 75 and 50, and poor for those below 50 points. The excellence rate $=($ Number of excellence + Number of good)/total $\times 100 \%$.

\section{Statistical methods:}

SPSS 20.0 software was utilized to analyze the data of this study. Measurement data were expressed in form of mean \pm standard deviation (SD) and compared to identify the difference between two groups by using $t$ test. Enumeration data were expressed by percentage, and compared by using the chi-square test. For theoretical frequency between 1 and 5, enumeration data were firstly rectified for test, while the count data were compared by using the rank sum test. $\mathrm{p}<0.05$ suggested that the difference had statistical significance.

\section{RESULTS AND DISCUSSION}

Operation time of patients in Group A was significantly shorter than that in Group B ( $\mathrm{p}<0.05)$, with less bleeding amount $(p<0.05$; Table 1$)$. At three mo after operation, patients in two groups had larger Bohler angle and Gissane angle as compared to these angles before operation $(p<0.05)$, while no significant difference was identified between these two groups ( $>0.05$; Table 2$)$.

\begin{tabular}{lccc}
$\begin{array}{l}\text { TABLE 1: COMPARISON } \\
\text { (MEAN }\end{array}$ & OF & THE & OPERATION \\
\hline Group & $\mathrm{n}$ & Operation time $(\mathrm{min})$ & Intraoperative bleeding amount $(\mathrm{mL})$ \\
\hline Group A & 30 & $60.32 \pm 10.19$ & $21.64 \pm 5.13$ \\
Group B & 30 & $91.04 \pm 13.85$ & $98.01 \pm 5.26$ \\
$\mathrm{t}$ & & 9.786 & 56.931 \\
$\mathrm{p}$ & & 0.000 & 0.000 \\
\hline
\end{tabular}


TABLE 2: COMPARISON OF THE CHANGES OF BÖHLER ANGLE AND GISSANE ANGLE BETWEEN TWO GROUPS (MEAN $\pm S D)$

\begin{tabular}{|c|c|c|c|c|c|}
\hline \multirow[b]{2}{*}{ Group } & \multirow{2}{*}{$\begin{array}{c}n \\
\text { (affected feet) }\end{array}$} & \multicolumn{2}{|c|}{ Böhler angle } & \multicolumn{2}{|c|}{ Gissane angle } \\
\hline & & Before operation & $\begin{array}{l}3 \text { mo after } \\
\text { operation }\end{array}$ & Before operation & $\begin{array}{l}3 \text { mo after } \\
\text { operation }\end{array}$ \\
\hline Group A & 32 & $14.19 \pm 2.47$ & $33.52 \pm 4.41^{\mathrm{a}}$ & $90.16 \pm 6.43$ & $129.45 \pm 9.83^{\circ}$ \\
\hline Group B & 31 & $14.08 \pm 2.35$ & $33.09 \pm 4.36^{a}$ & $90.11 \pm 6.37$ & $130.87 \pm 9.24^{\circ}$ \\
\hline $\mathrm{t}$ & & 0.181 & 0.389 & 0.031 & 0.590 \\
\hline $\mathrm{p}$ & & 0.857 & 0.699 & 0.975 & 0.557 \\
\hline
\end{tabular}

Note: ${ }^{\mathrm{p}} \mathrm{p}<0.05$ vs. the angles before operation

In two groups, no nerve damage was found. In Group A, there was 1 foot with the superficial infection at wound that was then recovered after medication, 2 feet with the traumatic subtalar arthritis that was recovered after anti-inflammation and physical therapy, with promising restoration of function and the incidence rate of complication was $9.38 \%(3 / 32)$. In Group B, there were 3 feet with superficial infection at wound and 1 foot with deep infection that were all recovered after the medication, 3 feet with the marginal necrosis of skin ( 2 feet that were recovered after the antiinflammatory treatment and physical therapy, 1 foot that was recovered after the subtalar joint fusion), and the incidence rate of complication was $32.26 \%(10 / 31)$. Thus, the incidence rate of complications in patients of Group A was significantly lower than that in Group B $\left(\chi^{2}=5.035 ; \pi=0.025\right)$.

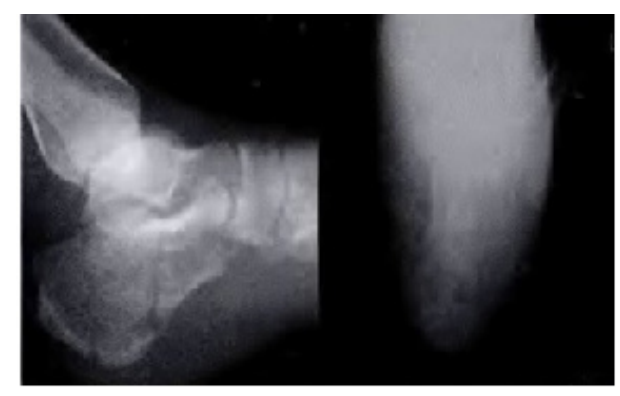

A: Prenperative $\mathrm{X}$-ray image showing the calcaneal fracture

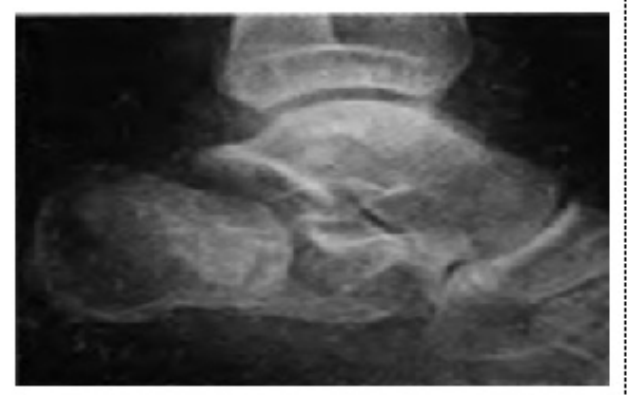

C: Prcoperattve $\mathrm{X}$ ray tmage showing the calcaneal fracturf
The postoperative follow-up ranged from 3 to 15 mo, with an average of $(10.15 \pm 2.67)$ mo. At the end of follow-up, no significant difference was shown in comparison of the excellence rates in foot recovery between two groups ( $>0.05$; Table 3 ).

Patient Zhou, male, 48 y old, with the Sanders III calcaneal fracture caused by falling injury, received the closed replacement and internal fixation of cannulated screws. Results of X-ray examination before and after operation are shown in fig. $1 \mathrm{~A}$ and $1 \mathrm{~B}$. Patient Li, male, 39 y old, with the Sanders calcaneal fracture caused by falling injury, received the open reduction and internal fixation of irregular steel plate. Results of X-ray examination before and after operation are shown in fig. $1 \mathrm{C}$ and $1 \mathrm{D}$.

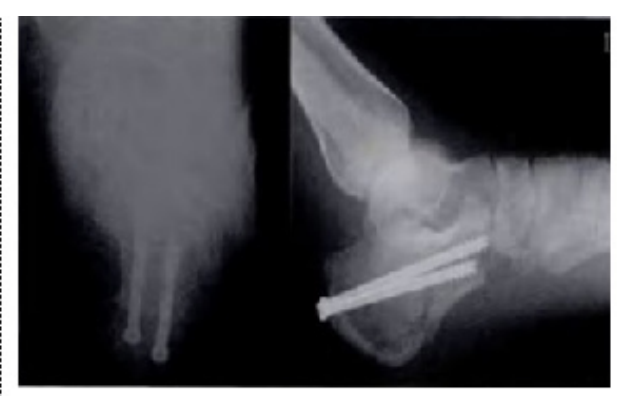

R: X-ray imnge nfter the clnsed replacement nnd internal fixation of cannulated screw

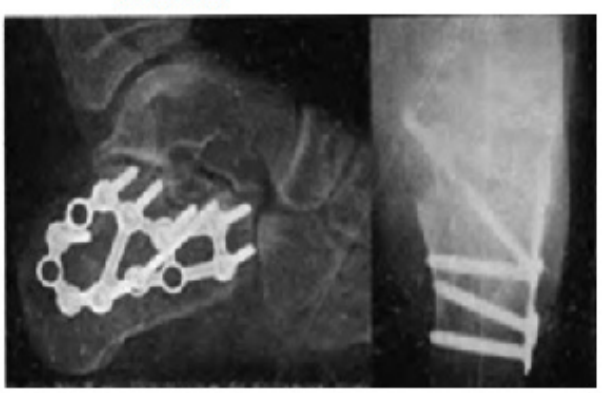

D: $\mathbf{X}$ ray image after the open reduction and internal fixation of irreguln $r$ steel plate

Fig. 1: Image of $X$-ray examination of patients 
www.ijpsonline.com

TABLE 3: COMPARISON OF THE RECOVERY OF FOOT FUNCTIONS [n (\%)]

\begin{tabular}{lllllll}
\hline Group & N of affected feet & Excellence & Good & Normal & Poor & Excellence rate \\
\hline Group A & 32 & $23(71.88)$ & $7(21.88)$ & $2(6.25)$ & $0(0.00)$ & $30(93.75)$ \\
Group B & 31 & $22(70.97)$ & $6(19.35)$ & $3(9.68)$ & $0(0.00)$ & $28(90.32)$ \\
Z/ $\chi^{2}$ & & 0.231 & & & & 0.253 \\
$\mathrm{p}$ & & 0.627 & & & & 0.615 \\
\hline
\end{tabular}

Calcaneal fracture is mainly caused by the vertical crash caused by the falling injury to the heel that may result in the shortening and widening of calcaneus, collapse and shifting of subtalar joint, and reduction or even disappearance of Bohler angle, and the changes in Gissane angles, and all these changes would further cause the deformity of foot and affect the regular function, deteriorating the life quality of patients ${ }^{[8-10]}$. Therefore, it is quite significant for the prognosis of fracture and restoration of joint function of patients to select the appropriate and effective methods to recover the normal appearance, para-positions of subtalar joints and normal Bohler angle and Gissane angle.

Results of this study showed that at 3 mo after operation, patients of two groups had significant enlargement in the Bohler angle and Gissane angle as compared to the angles before operation, while no significant difference was found in comparison between two groups. Besides, at the end of follow-up, the excellent rates of patients in function recovery of Group A and B were $93.75 \%$ and $90.32 \%$, showing no evident difference between two groups, suggesting that these two methods can both effectively promote the recovery of Bohler angle and Gissane angle, and the recovery of foot function. Close replacement and internal fixation of cannulated screw was performed under the C-shape arm to perform the reduction of fracture, with the evident efficacy, and the compressing screws can compress the fracture ends, which is conducive to the fracture reduction and reunion. In addition, the thread of screws is also proficient in compression, plus the anti-bending stress, which is also conducive the reduction of fracture and elimination of fracture space. Thus, it can sustain the reduction and fixation effectively to promote the restoration of foot function of patients. Current evidence ${ }^{[11,12]}$ shows that for the calcaneus fracture patients, closed reduction and internal fixation of cannulated screws gains promising outcomes, including the postoperative recovery of Bohler angle and Gissane angle, and the excellence rate of patients in recovery of foot function is $90 \%$, similar to the result of our work. For open reduction and internal fixation of irregular steel plate, the surgical incision can reach the bone surface to expose the calcaneal lateral side, collapsed subtalar joint surface and calcaneocuboid joint where, under the direct vision, Steinmann pin can be used to poke and tract to restore the calcaneal anatomic structure and sustain the smooth articular face, and for severe damage, artificial bone or iliac bone should be implanted to provide the strong support to prevent the collapse ${ }^{[13]}$. Additionally, fracture can be fixed by the plastic plate and the implantation of screws towards the sustentaculum tali can enhance the fixation, sustain the reduction, support the calcaneocuboid joint and contribute to the function exercise to promote the function recovery of affected foot $^{[14]}$. Existing data shows that open reduction and internal fixation of irregular steel plate gains promising efficacy in treatment of calcaneal fracture, and the excellence rate in foot function recovery is as high as $84.4 \%$.

The results of this study also showed that the operation time of patients in Group A was significantly shorter than that in Group B, with less intraoperative bleeding amount and lower incidence rate of postoperative complications, suggesting that the closed reduction and internal fixation of cannulated screws is excellent in the mild surgical trauma and reduction in the postoperative complications as compared to the open reduction and internal fixation of irregular steel plate. Moreover, closed reduction and internal fixation of cannulated screws brings about mild damage to the soft tissue, with no need of skin incision or massive dissection of periosteum or flaps of soft tissues surrounding the calcaneus and less to less bleeding amount in the operation, which can avoid the necrosis of $\operatorname{skin}^{[15]}$. Furthermore, the small wound and convenience in operation can enable the physicians to directly perform the reduction of calcaneal-talar joint and shorten the operation time ${ }^{[16]}$. Conversely, open reduction and internal fixation of irregular steel plate causes the severe damage to the soft tissue and increase the intraoperative bleeding amount, and the extensive dissection of soft tissue can increase the susceptibility of wound to the infection and flap necrosis. Furthermore, this method requires longer operation time ${ }^{[17]}$. 
To be concluded, closed replacement and internal fixation of cannulated screw and surgical open reduction and internal fixation of irregular steel plates can both gain promising outcomes in terms of the replacement and postoperative recovery of foot function for calcaneal fracture, while the former is excellent in the mild surgical trauma and low incidence of postoperative complications.

\section{Conflict of interests:}

The authors declared no conflicts of interest.

\section{REFERENCES}

1. Shariatzadeh H, Valiollahi B, Mohammadpour M, Haghbin M. Outcomes of Minimally-Invasive Sinus Tarsi Approach for the Treatment of Intra-articular Calcaneal Fracture. J Res Orthop Sci 2020;7:47-54.

2. Adams MR, Koury KL, Mistry JB, Braaksma W, Hwang JS, Firoozabadi R. Plantar Medial Avulsion Fragment Associated With Tongue-Type Calcaneus Fractures. Foot Ankle Int 2019;40:634-40

3. Cottom JM, Douthett SM, McConnell KK. Intraoperative Reduction Techniques for Surgical Management of Displaced Intra-Articular Calcaneal Fractures. Clin Podiatr Med Surg 2019;36:269-77.

4. Hordyk PJ, Fuerbringer BA, Roukis TS. Clinical Management of Acute, Closed Displaced Intra-Articular Calcaneal Fractures. Clin Podiatr Med Surg 2019;36:163-71.

5. Schepers T. Sinus Tarsi Approach with Screws-Only Fixation for Displaced Intra-Articular Calcaneal Fractures. Clin Podiatr Med Surg 2019;36:211-24.

6. Herrera-Pérez M, Oller-Boix A, Valderrabano V, GonzálezCasamayor S, Gutiérrez-Morales MJ, Guerra-Ferraz A, et al. Calcaneal fractures: controversies and consensus. Acta Ortop Mex 2018;32:172-81

7. Diacon AL, Kimmel LA, Hau RC, Gabbe BJ, Edwards ER. Outcomes of midfoot and hindfoot fractures in multitrauma patients. Injury 2019;50:558-63.

8. Shih JT, Kuo CL, Yeh TT, Shen HC, Pan RY, Wu CC. Modified Essex-Lopresti procedure with percutaneous calcaneoplasty for comminuted intra-articular calcaneal fractures: a retrospective case analysis. BMC Musculoskelet Disord 2018;19:77.
9. Wei N, Yuwen P, Liu W, Zhu Y, Chang W, Feng C, et al. Operative versus nonoperative treatment of displaced intraarticular calcaneal fractures: A meta-analysis of current evidence base. Medicine 2017;96:e9027.

10. Sanders R, Vaupel ZM, Erdogan M, Downes K. Operative treatment of displaced intraarticular calcaneal fractures: longterm (10-20 Years) results in 108 fractures using a prognostic CT classification. J Orthop Trauma 2014;28:551-63.

11. Aldridge JM, Easley M, Nunley JA. Open calcaneal fractures: results of operative treatment. J Orthop Trauma 2004;18:7-11.

12. Benirschke SK, Sangeorzan BJ. Extensive intraarticular fractures of the foot. Surgical management of calcaneal fractures. Clin Orthop Relat Res 1993:128-34.

13. Seat A, Seat C. Lateral Extensile Approach Versus Minimal Incision Approach for Open Reduction and Internal Fixation of Displaced Intra-articular Calcaneal Fractures: A Metaanalysis. J Foot Ankle Surg 2020;59:356-66.

14. Kline AJ, Anderson RB, Davis WH, Jones CP, Cohen BE. Minimally invasive technique versus an extensible lateral approach for intra-articular calcaneal fractures. Foot Ankle Int 2013;34:773-80.

15. Li LH, Guo YZ, Wang H, Sang QH, Zhang JZ, Liu Z, et al. Less wound complications of a sinus tarsi approach compared to an extended lateral approach for the treatment of displaced intraarticular calcaneal fracture: A randomized clinical trial in 64 patients. Medicine 2016;95:e4628.

16. Buckley R, Leighton R, Sanders D, Poon J, Coles CP, Stephen $\mathrm{D}$, et al. Open reduction and internal fixation compared with ORIF and primary subtalar arthrodesis for treatment of Sanders type IV calcaneal fractures: a randomized multicenter trial. J Orthop Trauma 2014;28:577-83.

17. Radnay CS, Clare MP, Sanders RW. Subtalar fusion after displaced intra-articular calcaneal fractures: does initial operative treatment matter? Surgical technique. J Bone Joint Surg Am 2010;92:32-43.

This is an open access article distributed under the terms of the Creative Commons Attribution-NonCommercial-ShareAlike 3.0 License, which allows others to remix, tweak, and build upon the work non-commercially, as long as the author is credited and the new creations are licensed under the identical terms

This article was originally published in a special issue,
"Trends in Therapeutic Management of Various Clinical
Conditions II" Indian J Pharm Sci 2021:83(2)Spl issue;155-160

